\title{
The Danish Testicular Cancer database
}

This article was published in the following Dove Press journal:

Clinical Epidemiology

25 October 2016

Number of times this article has been viewed

\author{
Gedske Daugaard' \\ Maria Gry Gundgaard Kier' \\ Mikkel Bandak' \\ Mette Saksø Mortensen' \\ Heidi Larsson ${ }^{2}$ \\ Mette Søgaard ${ }^{2}$ \\ Birgitte Groenkaer Toft ${ }^{3}$ \\ Birte Engvad ${ }^{4}$ \\ Mads Agerbæk ${ }^{5}$ \\ Niels Vilstrup Holm 6 \\ Jakob Lauritsen' \\ 'Department of Oncology 5073, \\ Copenhagen University Hospital, \\ Rigshospitalet, Copenhagen, \\ 2Department of Clinical Epidemiology, \\ Aarhus University Hospital, \\ Aarhus, ${ }^{3}$ Department of Pathology, \\ Copenhagen University Hospital, \\ Rigshospitalet, Copenhagen, \\ ${ }^{4}$ Department of Pathology, Odense \\ University Hospital, Odense, \\ ${ }^{5}$ Department of Oncology, Aarhus \\ University Hospital, Aarhus, \\ ${ }^{6}$ Department of Oncology, Odense \\ University Hospital, Odense, Denmark
}

Correspondence: Gedske Daugaard

Department of Oncology 5073,

Copenhagen University Hospital,

Rigshospitalet, Blegdamsvej 9,

Copenhagen, Denmark

Tel +45 35454677

Fax +4535456966

Email kirsten.gedske.daugaard@regionh.dk
Aim: The nationwide Danish Testicular Cancer database consists of a retrospective research database (DaTeCa database) and a prospective clinical database (Danish Multidisciplinary Cancer Group [DMCG] DaTeCa database). The aim is to improve the quality of care for patients with testicular cancer (TC) in Denmark, that is, by identifying risk factors for relapse, toxicity related to treatment, and focusing on late effects.

Study population: All Danish male patients with a histologically verified germ cell cancer diagnosis in the Danish Pathology Registry are included in the DaTeCa databases. Data collection has been performed from 1984 to 2007 and from 2013 onward, respectively.

Main variables and descriptive data: The retrospective $\mathrm{DaTeCa}$ database contains detailed information with more than 300 variables related to histology, stage, treatment, relapses, pathology, tumor markers, kidney function, lung function, etc. A questionnaire related to late effects has been conducted, which includes questions regarding social relationships, life situation, general health status, family background, diseases, symptoms, use of medication, marital status, psychosocial issues, fertility, and sexuality. TC survivors alive on October 2014 were invited to fill in this questionnaire including 160 validated questions. Collection of questionnaires is still ongoing. A biobank including blood/sputum samples for future genetic analyses has been established. Both samples related to DaTeCa and DMCG DaTeCa database are included. The prospective DMCG DaTeCa database includes variables regarding histology, stage, prognostic group, and treatment.

Conclusion: The DMCG DaTeCa database has existed since 2013 and is a young clinical database. It is necessary to extend the data collection in the prospective database in order to answer quality-related questions. Data from the retrospective database will be added to the prospective data. This will result in a large and very comprehensive database for future studies on TC patients.

Keywords: testis cancer, clinical indicators, database research, DaTeCa, DMCG DaTeCa database, hypogonadism

\section{Aim of database}

Testicular cancer (TC) is a rare disease, with $\sim 300$ cases per year in Denmark. Nonetheless, TC is the most common cancer in men aged 20-40 years. Today, it represents the most curable solid tumor with a 10-year survival of $90 \%-95 \%{ }^{1}{ }^{1}$

With the high cure rate and young age at diagnosis, posttreatment morbidity plays a central role. Previous studies have shown an increased risk of secondary cancers, and conditions including heart disease, diabetes, hypogonadism, decreased fertility, and psychosocial problems. ${ }^{2}$ Data regarding risk factors for long-term side effects submit your manuscript | www.dovepress.com

Dovepress

http://dx.doi.org/10.2147/CLEP.S99493
Clinical Epidemiology 2016:8 703-707 (Thematic series on clinical quality databases in Denmark) 703 (c) (1) (5) 2016 Daugaard et al. This work is published and licensed by Dove Medical Press Limited. The full terms of this license are available at https://www.dovepress.com/ cc) terms.php and incorporate the Creative Commons Attribution - Non Commercial (unported, v3.0) License (http://(creativecommons.org//icenses/by-nc/3.0/). By accessing the work you hereby accept the Terms. Non-commercial uses of the work are permitted without any further permission from Dove Medical Press Limited, provided the work is properly attributed. For permission for commercial use of this work, please see paragraphs 4.2 and 5 of our Terms (https://www.dovepress.com/terms.php). 
of treatment are modest, and the precise risk of the various late side effects remains unresolved.

In Denmark, treatment of TC after orchiectomy has been increasingly centralized over the years, and is now performed only at three university hospitals. A common treatment and follow-up strategy has been developed across these centers (Table 1). National guidelines for treatment have been set by a multidisciplinary group. This group consists of representatives from all attending departments and from specialties relevant in the treatment of TC (www.ducg.dk). We have a large retrospective and a smaller prospective database, which will be merged in the coming years.

The overall aim of both databases is to improve the quality of care for patients with TC in Denmark. Several important issues related to treatment and survivorship of TC patients such as prognostic factors for relapse, treatment, follow-up, and late effects may be described through analyses of data from the clinical databases. So far, the focus has been on the following areas:

- Analyses of quality indicators, benchmarking, quality audit, and feedback to departments.

- Prognostic factors for relapse in stage I seminoma and nonseminoma patients. ${ }^{3,4}$

- Rational follow-up of stage I patients in a surveillance program. ${ }^{3,4}$

- Screening for contralateral carcinoma in situ testis. ${ }^{5}$

- National treatment results for patients with metastatic disease including reanalysis of international risk factors published in $1997 .{ }^{6}$

- Patients treated with more than one line of therapy for disseminated disease. ${ }^{7}$

- Second primary cancer and cause of death.

Table I Testicular cancer - diagnosis and treatment

General staging:
Computed tomography scan and tumor markers ( $\alpha$-fetoprotein, human
chorionic gonadotropin, lactate dehydrogenase)
Stage I:
Tumor in one or both testicles
Treatment
All patients followed on a surveillance program
Metastatic disease
Divided according to prognostic factors into a good, intermediate, and
poor risk group
Treatment
Radiotherapy for stage Ila and llb seminoma with lymph nodes $<3 \mathrm{~cm}$.
Three or four cycles of cisplatin, etoposide, and bleomycin for all other
patients
Risk factors
Histology, tumor markers, metastases outside lung and lymph nodes,
extragonadal tumors in mediastinum of nonseminoma origin

- Long-term morbidity in the form of cardiovascular disease, metabolic syndrome, neurotoxicity, nephrotoxicity, pulmonary toxicity, hypogonadism, decreased fertility, and psychosocial problems. ${ }^{89}$

\section{Study population}

There are two TC databases in Denmark. Initially, the retrospective Danish Testicular Cancer (DaTeCa) database was constructed (Danish data protection agency J.nr. 2012-410751). This is a research database comprising $\sim 6,000$ Danish male patients with TC diagnosed from January 1, 1984 to December 31, 2007. Subsequently, the prospective Danish Multidisciplinary Cancer Group (DMCG) DaTeCa database was initiated in 2013 with prospective data collection of key clinical variables. The databases include cancers of both gonadal and extragonadal origin.

Patients in the retrospective DaTeCa database have been identified through the Danish National Patient Registry ${ }^{10}$ and hospital files. The diagnoses have been confirmed through manual review of pathology reports. The prospective DMCG DaTeCa database includes all incident TCs of both gonadal and extragonadal origin in Denmark recorded in the Danish National Patient Registry and/or the Danish Pathology Registry ${ }^{11}$ from 2013 onward. At present, $\sim 650$ patients have been included in the database, with 300 new cases added every year. Up to now, the main focus has been on optimizing the validity of the algorithm for patient identification in the central registries, and validation of pathology data from three larger hospitals diagnosing and treating patients have revealed $100 \%$ coverage of histologically verified cancers. Further validation will ensure an even higher validity and completeness of data in the future.

\section{Main variables}

The retrospective DaTeCa database contains detailed information with more than 300 variables related to histology, stage, treatment, relapses, pathology, tumor markers, kidney function, lung function, etc. Information about cause of death has been obtained from the Danish Registry of Causes of Death and cross-checked against journal files. Through linkage with national central registries, medical history after 5 years of standard follow-up program has been obtained, including vital status. A questionnaire related to late effects has been prepared, which includes issues on social relationships, life situation, general health status, family background, diseases, symptoms, use of medication, marital status, psychosocial issues, fertility, and sexuality. Patients have been asked to fill this questionnaire, and to deliver relevant blood/sputum tests 
to a biobank for future genetic analyses. These data will be included in the retrospective database. In the longer term, the same data will be collected in the prospective database.

The prospective DMCG DaTeCa database includes variables regarding histology, stage, prognostic group, and treatment. These variables are obtained by data linkage to the Danish Pathology Registry (eg, histology and stage), the Danish National Patient Register (eg, medical history, surgical procedures, chemotherapy, and radiation therapy), and the Civil Registration System (data on vital status and migration). Additional key clinical variables are registered online in a web-based form by the treating clinicians at the oncological departments. These online registration forms are partly uniform for the five uro-oncological databases in Denmark. In 2013 and 2014, $98 \%$ of all newly diagnosed patients identified in the central registries have had an online registration form filled in.

Seven clinical quality indicators (four result indicators and three process indicators) are derived from the prospective database in order to monitor and improve the quality of care for patients with TC. These quality indicators include both indicators describing remission after treatment (indicator $1 \mathrm{a}-\mathrm{c}$ ), surgery (indicator 2), occurrence of relapse (indicator $3 \mathrm{a}-\mathrm{b}, 4)$, prognosis, and the completeness of the pathological coding (indicator 5-7) (Table 2).
The relevant pathological codes used to identify prognosis and risk of relapse are important, and from 2013 to 2014, a significant improvement of coding practice was observed. ${ }^{12}$ With continuous attention, also regarding coding of other clinical and paraclinical parameters, we found it possible to meet the expected quality standards.

\section{Follow-up}

Patients are followed by uniform national follow-up schedules, usually for 5 years. After that, survival and possible relapse can be followed in national registries.

\section{Examples of research}

Patients with stage I TCs are followed for 5 years in a surveillance program. It is demanded that follow-up programs are effective and relevant for the survival of the patient. We have used the retrospective database to obtain detailed information about relapses including risk factors for relapse both in seminoma and nonseminoma patients. ${ }^{3,4}$ Furthermore, we have been able to suggest a timetable for follow-up.

The relapse rate after orchiectomy in stage I nonseminoma was $30.6 \%$ after 5 years. Presence of vascular invasion together with embryonal carcinoma and rete testis invasion in the testicular primary identified a group of patients with a relapse risk of $50 \%$. Without risk factors, the relapse

Table 2 Clinical quality indicators in the prospective DaTeCa database and indicator fulfillment in 2013 and 2014

\begin{tabular}{|c|c|c|c|c|}
\hline \multirow[b]{3}{*}{ Indicator } & \multirow[b]{3}{*}{ Standard (\%) } & \multirow{3}{*}{$\frac{\text { Unknown }}{\%}$} & \multicolumn{2}{|c|}{ Indicator fulfillment \% } \\
\hline & & & \multicolumn{2}{|l|}{$95 \% \mathrm{Cl}$} \\
\hline & & & 2014 & 2013 \\
\hline $\begin{array}{l}\text { Indicator Ia: CR after chemotherapy. Proportion of patients } \\
\text { with good prognosis, with CR after chemotherapy }\end{array}$ & $>95$ & 21.3 & 95 (82-99) & $100(92-100)$ \\
\hline $\begin{array}{l}\text { Indicator Ib: CR after chemotherapy. Proportion of patients } \\
\text { with intermediate prognosis, with CR after chemotherapy }\end{array}$ & $>80$ & 16.7 & $90(55-100)$ & $82(48-98)$ \\
\hline $\begin{array}{l}\text { Indicator I c: CR after chemotherapy. Proportion of patients } \\
\text { with poor prognosis, with CR after chemotherapy }\end{array}$ & $>60$ & 71.4 & $100(16-100)$ & $60(15-95)$ \\
\hline $\begin{array}{l}\text { Indicator 2: Secondary surgery. Proportion of patients with } \\
\text { secondary surgery for residual tumor }>1 \mathrm{~cm} \text { and nonseminoma }\end{array}$ & $>90$ & 0.0 & $93(68-100)$ & $100(82-100)$ \\
\hline $\begin{array}{l}\text { Indicator 3a: Relapse. Proportion of patients with relapse } \\
\text { after stage I nonseminoma followed on a surveillance program }\end{array}$ & $<30$ & 0.0 & & $16(8-27)$ \\
\hline $\begin{array}{l}\text { Indicator 3b: Relapse. Number of patients with relapse after } \\
\text { stage I seminoma followed on a surveillance program }\end{array}$ & $<20$ & 0.0 & & $5(2-10)$ \\
\hline $\begin{array}{l}\text { Indicator 4: Relapse. Proportion of patients with relapse after } \\
\text { first-line treatment for disseminated disease }\end{array}$ & $<15$ & 0.0 & & $6(2-15)$ \\
\hline $\begin{array}{l}\text { Indicator 6: Pathology. Proportion of newly diagnosed patients } \\
\text { with coding for PT stage }\end{array}$ & $>95$ & & 95 (92-97) & $88(84-92)$ \\
\hline $\begin{array}{l}\text { Indicator 7: Pathology. Proportion of newly diagnosed patients } \\
\text { with coding for vascular invasion: "present," "not present," or } \\
\text { "evaluation not possible" }\end{array}$ & $>95$ & & $69(63-74)$ & $56(50-62)$ \\
\hline $\begin{array}{l}\text { Indicator 8: Pathology. Proportion of newly diagnosed patients } \\
\text { with SNOMED coding for tumor diameter }\end{array}$ & $>95$ & & $73(68-78)$ & $60(54-66)$ \\
\hline
\end{tabular}

Abbreviations: DaTeCa database, Danish Testicular Cancer database; Cl, confidence interval; CR, complete remission; pT, pathological tumor; SNOMED, Systematized Nomenclature of Medicine. 
risk was $12 \%$. With a median follow-up of 15 years in the seminoma group, we found the relapse risk was $18.9 \%$. Tumor size was an important risk factor for relapse. Either vascular invasion or invasion of epididymis was significant, if the other factor was excluded from analysis. For a patient with seminoma, and characterized by a tumor diameter of $8 \mathrm{~cm}$ and vascular invasion, the 5-year risk of recurrence was $40 \%$.

Preventive treatment of recurrences of high-risk stage I patients with chemotherapy or radiation therapy is practiced at many centers worldwide. Based on our data, the risk factors underlying the selection of patients for treatment is incorrect. The newly defined risk factors identified through use of the DaTeCa database needs to be confirmed prospectively before deciding on adjuvant treatment in high-risk patients. As Denmark is one of the few countries worldwide in which all stage I patients are followed with surveillance, the confirmation of risk factors will be performed on patients in the prospective DMCG DaTeCa database. Data completeness concerning histology in the prospective DMCG database is expected to be better compared with the retrospective database, and we aim to validate the risk factors mentioned above and possibly identify additional factors.

In 2015, the first annual report from the prospective DMCG DaTeCa database was published online at www. Sundhed.dk. The report contained detailed analyses on indicator fulfillment in 2013 and 2014 at the hospital level, regional level, and national level.

\section{Administrative issues and funding}

The retrospective DaTeCa database was created as a research database and analyses from this database have received support from various funding and already created several publications. $^{3-5,7-9}$

The prospective DMCG DaTeCa database is under the auspices of the Danish Multidisciplinary Cancer Groups (DMCG.dk), which is an umbrella organization comprising 24 national cancer groups and clinical databases in Denmark. ${ }^{13}$ The urological cancer groups and databases are further organized under the subgroup for Danish Urological Cancer Groups. ${ }^{14}$

The prospective DMCG DaTeCa database is publicly funded by the Danish Regions ${ }^{15}$ and under the administration of the Danish Clinical Registries (RKKP). ${ }^{16}$ Technical support and data management is provided by the Registry Support Centres of Clinical Quality and Health Informatics (West) (KCKS-Vest) ${ }^{17}$ and epidemiological and statistical support to the annual reports is provided by the Registry Support Centre of Epidemiology and Biostatistics (North)
(KCEB-Nord). ${ }^{18}$ The Danish Clinical Registries (RKKP) is a nationwide initiative for monitoring and improving the quality of clinical care in Denmark.

\section{Conclusion}

The retrospective $\mathrm{DaTeCa}$ database is the most detailed database concerning TC worldwide. The strength of the database is the complete and detailed information relating to treatment and that the treatment is consistent with today's international standards, both in terms of stage I disease and chemotherapy for metastatic disease. We have focused on patients treated between 1984 and 2007 to ensure at least 5-year follow-up of all patients. It is the intention to use the prospective DMCG DaTeCa database to validate findings in the retrospective database and to expand the database to include a larger amount of data from public registries. The quality indicators will be used to improve quality of treatment. It is a challenge to identify and ensure the use of correct diagnosis codes so that all relevant patients are included in the database. Furthermore, data from the retrospective database will be added to the prospective data. This will result in a large and comprehensive database for future studies on TC patients. In the long term, the main variables in the prospective database should be expanded to cover results from relevant laboratory analyses, and results from specialized tests, that is, single nucleotide polymorphism analysis.

Data regarding the factors leading to long-term side effects of treatment are scarce. The introduction of new molecular testing methods allows us to use these tools to identify patients at high risk for therapy-related complications and thus the opportunity to develop risk-adapted screening and intervention strategies. The available database along with the biobank is optimal for this purpose.

\section{Acknowledgments}

This paper was funded by the Program for Clinical Research Infrastructure established by the Lundbeck Foundation and the Novo Nordisk Foundation and administered by the Danish Regions.

\section{Disclosure}

The authors report no conflicts of interest in this work.

\section{References}

1. Verdecchia A, Francisci S, Brenner H, et al. Recent cancer survival in Europe: a 2000-2002 period analysis of EUROCARE-4 data. Lancet Oncol. 2007;8:784-796.

2. Travis LB, Beard C, Allan JM, et al. Testicular cancer survivorship: research strategies and recommendations. J Natl Cancer Inst. 2010; 102:1114-1130. 
3. Mortensen MS, Lauritsen J, Gundgaard MG, et al. A nationwide cohort study of stage I seminoma patients followed on a surveillance program. Eur Urol. 2014;66(6):1172-1178.

4. Daugaard G, Gundgaard MG, Mortensen MS, et al. Surveillance for stage I non-seminomatous testicular cancer - outcomes and long-term follow-up in a population based cohort. J Clin Oncol. 2014;32(34): 3817-3823.

5. Kier MG, Lauritsen J, Almstrup K, et al. Screening for contralateral carcinoma in situ testis in patients with testicular cancer: a population based study. DaTeCa 03 study. Ann Oncol. 2015;26(4):737-742.

6. International Germ Cell Cancer Collaborative Group: International Germ Cell Consensus Classification: a prognostic factor-based staging system for metastatic germ cell cancer. J Clin Oncol. 1997;15:594-596.

7. Lauritsen J, Kier MGG, Mortensen MS, et al. Germ cell cancer and multiple relapses - toxicity and survival. J Clin Oncol. 33:3116-3123.

8. Bandak M, Aksglaede L, Juul A, Rørth M, Daugaard G. The pituitaryLeydig cell axis before and after orchiectomy in patients with stage I testicular cancer. Eur J Cancer. 2011;47(17):2585-2591.

9. Lauritsen J, Mortensen MS, Kier MG, et al. Renal impairment and late toxicity in germ-cell cancer survivors. Ann Oncol. 2015; 26(1):173-178.

10. Schmidt M, Schmidt SA, Sandegaard JL, Ehrenstein V, Pedersen L, Sorensen HT. The Danish National Patient Registry: a review of content, data quality, and research potential. Clin Epidemiol. 2015;7:449-490.
11. Bjerregaard B, Larsen OB. The Danish Pathology Register. Scand J Public Health. 2011;39(7 Suppl):72-74.

12. Kliniske databaser [homepage on the Internet]. Sundhed.dk [updated 2014 Dec 31]. Available from: https:/www.sundhed.dk/content/ cms/86/15686_dateca-\%C3\%A5rsrapport_2014_kommenteret_ 20150328.pdf. Accessed February 15, 2016.

13. Danish Multidisciplinary Cancer Groups (DMCG.dk). Available from: http://dmcg.dk/. Accessed August 1, 2015.

14. Danish Urological Cancer Groups (DUCG). Available from: http:// ducg.dk/. Accessed August 1, 2015.

15. Danish Regions. Available from: http://www.regioner.dk/. Accessed August 1, 2015.

16. Danish Quality Improvement Program (RKKP). Available from: http:// www.rkkp.dk/. Accessed August 1, 2015.

17. Competence Centre for Health Quality and Informatics (KSKS-Vest). Available from: http://www.kcks-vest.dk/kliniske-kvalitetsdatabaser/. Accessed August 1, 2015.

18. Competence Centre for Clinical Epidemiology and Biostatistics, North (KCEB-Nord). Available from: http://www.kea.au.dk/en/ClinicalQuality/CentreClinEpiBiostatNorth.html. Accessed August 1, 2015.
Clinical Epidemiology

\section{Publish your work in this journal}

Clinical Epidemiology is an international, peer-reviewed, open access, online journal focusing on disease and drug epidemiology, identification of risk factors and screening procedures to develop optimal preventative initiatives and programs. Specific topics include: diagnosis, prognosis, treatment, screening, prevention, risk factor modification,

Submit your manuscript here: http://www.dovepress.com/clinical-epidemiology-journa

\section{Dovepress}

systematic reviews, risk \& safety of medical interventions, epidemiology \& biostatistical methods, and evaluation of guidelines, translational medicine, health policies \& economic evaluations. The manuscript management system is completely online and includes a very quick and fair peer-review system, which is all easy to use. 